\title{
Algoritmo de optimización para la detección en un sistema $\varphi$-OTDR basado en un láser DFB encadenado a través de anillo resonador de fibra óptica
}

\author{
C.A. López-Mercado ${ }^{a}$, V.V. Spirin ${ }^{a}$, J.L. Bueno Escobedo ${ }^{a}$, J. Jason ${ }^{b}$, and A.A. Fotiadi ${ }^{b, c, d}$ \\ ${ }^{a}$ Centro de Investigación Científica y de Educación Superior de Ensenada, \\ Carretera Ensenada-Tijuana No. 3918, Zona Playitas, 22860 Ensenada, B.C. México. \\ ${ }^{b}$ University of Mons, Boulevard Dolez 31, 7000 Mons, Belgium. \\ ${ }^{c}$ Ulyanovsk State University, 42 Leo Tolstoy Street, Ulyanovsk, 432970, Russia. \\ ${ }^{d}$ Ioffe Physico-Technical Institute of the RAS, 26 Polytekhnicheskaya Street, St. Petersburg 194021, Russia.
}

Received 10 March 2020; accepted 16 May 2020

Presentamos un algoritmo complejo de promediación diferencial espacial-temporal que incrementa la capacidad de detección de un sistema de reflectrometría óptica en el dominio del tiempo sensible a la fase ( $\varphi-$ OTDR), el cual emplea un láser DFB estándar de telecomunicaciones para mediciones distribuidas en la fibra de prueba. El láser DFB es encadenado por auto-inyección a través de un anillo resonador de fibra óptica de polarización preservada, que genera una coherencia y estabilidad frecuencial de la emisión del láser para mediciones precisas. El algoritmo de promediación presentado es capaz de mejorar la SNR en 5-6 dB, incrementando significativamente la capacidad de detección del sistema $\varphi$-OTDR. Se demuestra la localización de perturbaciones a la frecuencia de $815 \mathrm{~Hz}$ en una distancia aproximada de 850 m con una precisión de $20 \mathrm{~m}$.

Descriptores: OTDR sensible a la fase; encadenamiento por auto-inyección; anillo resonador de fibra óptica.

We present a new spatial-temporal differential averaging algorithm that improves the capacity of our phase-sensitive optical time-domain reflectometry $(\varphi-$ OTDR) system to vibration detection. Specifically, the system employs the standard DFB laser self-injection locked through an external feedback loop comprising a PM fiber-optic ring resonator that enhances coherence and provides enough frequency stability to the laser operation. The reported algorithm is responsible for 5-6 dB increase of the SNR thus improving the system ability to detect vibrations. We demonstrate the vibrations with the frequency of $\sim 815 \mathrm{~Hz}$ at the distance of $\sim 850 \mathrm{~m}$ localized with a spatial resolution of $\sim 20 \mathrm{~m}$.

Keywords: Phase sensitive OTDR; self-injection locking; optical fiber ring resonator.

PACS: 42.81.Pa; 42.55.Px; 42.65.Es

\section{Introducción}

La técnica de reflectrometría óptica en el dominio del tiempo sensible a la fase ( $\varphi$-OTDR) es ampliamente estudiada por sus diversas aplicaciones como en la seguridad perimetral, seguridad en ductos de hidrocarburos, seguridad en vías férreas y en monitoreo estructural de puentes y presas, etc. [1-6]. El sistema $\varphi$-OTDR utiliza la dispersión de Rayleigh de pulsos de luz coherente que viajan en una fibra óptica monomodo para la detección y localización de vibraciones, la fibra óptica es empleada como elemento sensitivo y su longitud puede ser de varios kilómetros [1-6]. Las investigaciones recientes se centran en el mejoramiento del desempeño de estos sistemas mediante: esquemas de demodulación $I / Q$ y detección coherente homodina [6,7], el uso de diferentes formas especiales del pulso de prueba [8,9], pulsos de frecuencia lineal modulada [10], esquemas de demodulación multifrecuenciales y compensaciones del camino óptico [11], aplicación de señal de prueba de pulso dual [12], entre otras. Sin embargo, el uso de equipos más complejos y sofisticados incrementan el costo y la simplicidad de los sistemas $\varphi$-OTDR.

El algoritmo de procesamiento del sistema $\varphi$-OTDR busca cambios en la amplitud de la señal retrodispersada de Rayleigh entre trazas de pulsos consecutivos. La variación en la amplitud de la señal es un indicio de la presencia de
DOI: https://doi.org/10.31349/RevMexFis.66.637

una perturbación dinámica en alguna posición a lo largo de la fibra óptica de prueba. Por lo tanto, para una operación apropiada del sistema $\varphi$-OTDR, el desplazamiento de la frecuencia óptica entre dos pulsos consecutivos debe ser lo suficientemente baja para mantener un patrón de interferencia de retrodispersión de Rayleigh estable, y así la diferencia entre trazas consecutivas debe ser mínima para una fibra sin perturbación. Usualmente para mediciones de grandes distancias se requiere de una fuente de luz láser de alta coherencia con algunos $\mathrm{kHz}$ de ancho de línea espectral y un desplazamiento en frecuencia menor a $1 \mathrm{MHz} / \mathrm{min}$ [2]. Estos láseres de alta coherencia son de costos elevados y su diseño contribuye mayormente al costo total del sistema $\varphi$-OTDR.

Por otra parte, se ha estudiado ampliamente que el encadenamiento por auto- inyección de láseres DFB estándar de telecomunicaciones puede mejorar considerablemente su desempeño espectral [13,14]. En trabajos anteriores hemos demostrado el estrechamiento substancial del ancho de línea del láser por medio de una retroalimentación selectiva del espectro a través de un anillo resonador de fibra óptica (ARFO) desarrollado con componentes pasivos comunes de telecomunicaciones de bajo costo [15-17]. El láser DFB se encadena a un modo del ARFO cuando la señal de retroalimentación hacia la cavidad del láser es lo suficientemente potente para alcanzar la condición de encadenamiento. Esta solución completamente óptica y de bajo costo permite alcanzar un ancho 
de línea del láser de algunos kHz. Sin embargo, el régimen de frecuencia única usualmente es observado durante intervalos que son interrumpidos por saltos de modo de corta duración [15-17]. En trabajos anteriores se ha demostrado que el empleo de un algoritmo de promediación incrementa la relación señal a ruido de la detección de la perturbación [18].

En este trabajo se propone un algoritmo complejo de promediación espacial- temporal para la optimización de la detección de perturbaciones implementado en un sistema $\varphi-$ OTDR, que es novedoso para nuestro conocimiento. El algoritmo es adaptado a un sistema $\varphi$-OTDR que utiliza un láser DFB de bajo costo encadenado a través de un ARFO de polarización preservada PM optimizado para detección y localización de perturbaciones en la fibra de prueba. Demostrando un incremento en la relación señal a ruido SNR en 5-6 dB, aumentando significativamente la capacidad de detección de una perturbación con el sistema $\varphi$-OTDR propuesto.

\section{Configuración experimental}

El esquema experimental del sistema $\varphi-$ OTDR con el láser DFB encadenado por auto-inyección (LEI-DFB) es presentado en la Fig. 1. El láser DFB convencional opera en una longitud de onda de $\sim 1546 \mathrm{~nm}$ con una potencia de $5 \mathrm{~mW}$ y es encadenado en el régimen de acoplamiento crítico por medio del ARFO [15]. En este trabajo optimizamos el esquema experimental presentado en [18]. Se aumentó el factor de calidad del ARFO mediante el uso dos acopladores 99/1 dentro de ARFO y se incrementó su longitud hasta $4 \mathrm{~m}$ de fibra de polarización preservada, eliminando completamente el salto de modo asociado con la inestabilidad de la polarización entre dos estados de polarización ortogonales [19]. Como resultado de las modificaciones realizadas, logramos reducir el ancho de línea del láser DFB encadenado hasta 7.4 kHz. Así como la manipulación de la potencia de la retroalimentación al láser DFB para controlar el rango de auto encadenamiento [20], incrementando significativamente la estabilidad temporal del sensor en 2-3 veces. Además, estas modificaciones nos permiten registrar el momento cuando se presenta un salto de modo, y utilizar esta información en el procesamiento de la señal.

La emisión del LEI-DFB es amplificada por un amplificador de fibra dopada con Erbio (EDFA) y es modulada en

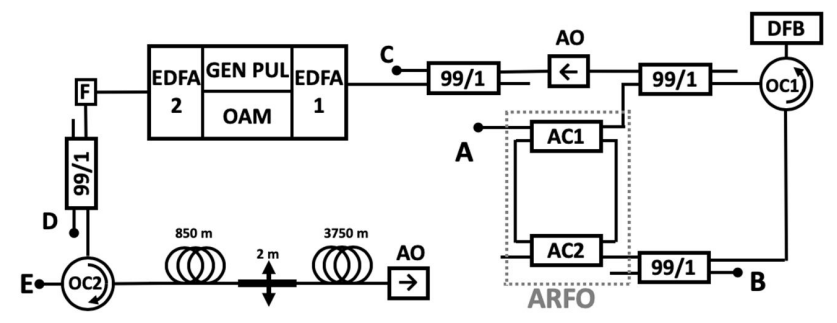

FIGURA 1. Esquema experimental para la detección de la perturbación generada en la fibra de prueba, ARFO-anillo resonador de fibra óptica, OC-circulador óptico, F-filtro pasa-banda, OAMmodulador acusto-óptico, AO-aislador óptico. amplitud por el modulador acusto-óptico OAM. El generador de pulsos eléctricos es el encargado de producir los pulsos cuadrados de $100 \mathrm{~ns}$ con una tasa de repetición de $20.3 \mathrm{kHz}$. Los pulsos ópticos son amplificados por un segundo EDFA y pasados por un filtro óptico pasa-banda de $1 \mathrm{~nm}$ para reducir la emisión espontanea generada en las etapas de amplificación. La potencia óptica en la salida del filtro es de $0.5 \mathrm{~mW}$ y la potencia pico de $95 \mathrm{~mW}$. La señal filtrada es dividida en dos brazos por un acoplador óptico de 99/1, donde el brazo correspondiente al $1 \%$ es utilizado para la sincronización de la tarjeta de adquisición de datos en el puerto $\mathrm{D}$, y el otro brazo del $99 \%$ es enviado a la fibra de prueba a través del circulador óptico OC2.

El puerto $E$ es utilizado para la detección y almacenamiento de la retrodispersión de Rayleigh generada en la fibra de prueba, utilizando un fotodetector de $5 \mathrm{GHz}$. La fibra de prueba es fibra óptica estándar de telecomunicaciones SMF-28e con una longitud de $\sim 4.5 \mathrm{~km}$ y cuenta con un aislador óptico en su extremo terminal, para evitar las reflexiones del fin de la fibra. La perturbación se induce en una sección de 2 metros de la fibra de prueba, esta es extendida por un material flexible al cual se le aplican movimientos mecánicos en dirección transversal con un piezoeléctrico a una frecuencia de $815 \mathrm{~Hz}$ de forma sinusoidal.

Para reducir las posibles causas que pueden generar un salto de modo, realizamos una estabilización precisa de la corriente y la temperatura de operación del láser DFB en los niveles clave que presentan un amplio intervalo del encadenamiento de la frecuencia del láser con el modo del ARFO [20]. El láser DFB fue operado con una corriente de alimentación de $50 \mathrm{~mA}$ ( 5 veces mayor al umbral) a temperatura ambiente de $25^{\circ} \mathrm{C}$, logrando una estabilidad con una precisión del $0.3 \%$ en ambos parámetros. En el trabajo experimental controlamos meticulosamente la influencia del ruido de la emisión espontanea de los EDFAs en el láser encadenado DFB con aisladores ópticos y el filtro estrecho pasa-banda, ya que este ruido degenera el funcionamiento del LEI-DFB [20]. Para minimizar las perturbaciones de temperatura y ambientales, el LEI-DFB y el ARFO fueron colocados dentro de una caja cerrada herméticamente con un aislamiento especial.

\section{Resultados experimentales y discusión}

El funcionamiento del ARFO es análogo a un interferómetro Fabry-Perot, en donde los puertos A y B (Fig. 1) representan al puerto reflejado y transmitido, respectivamente, y dependiendo de la condición de resonancia, la señal de entrada puede ser reflejada o transmitida a través del ARFO [15]. Cuando la frecuencia del láser DFB se encuentra en resonancia con un modo del ARFO, la potencia de entrada pasa libremente por el ARFO y regresa a la cavidad del láser consiguiendo la condición de encadenamiento, la cual provoca un drástico cambio en el comportamiento temporal de la potencia trasmitida en el puerto B (Fig. 1). La estabilidad de la potencia en el puerto B se presenta por intervalos relativamente largos, los 

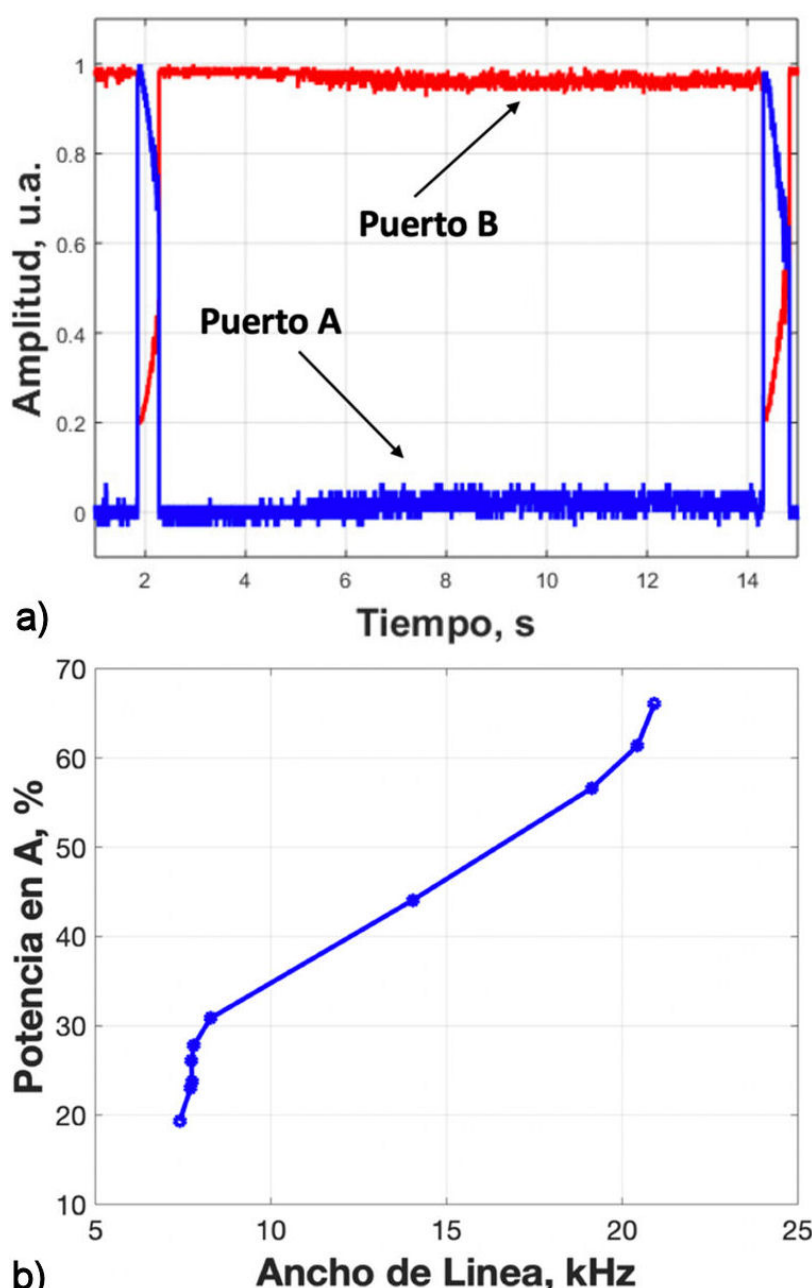

Figura 2. a) Trazas de osciloscopio de los puertos transmitido (puerto B) y reflejado (puerto A) del ARFO, b) ancho de línea del LEI-DFB vs la potencia normalizada del puerto reflejado.

cuales son interrumpidos por saltos de corto tiempo asociados con saltos de modo. Los periodos de estabilidad de 10-20 segundos son comunes en los láseres estabilizados por medio de un ARFO de fibra PM.

En la Fig. 2a) se presentan los trazos típicos de osciloscopio del comportamiento temporal de la potencia reflejada $y$ trasmitida del ARFO en los puertos A y B, respectivamente. Cuando el láser DFB no se encuentra encadenado a un modo o se presenta un salto de modo, toda la potencia de entrada al ARFO es desviada al puerto reflejado, lo que genera una supresión de potencia en el puerto transmitido. La supresión de potencia de la retroalimentación genera una disminución del nivel de encadenamiento del láser DFB, incrementando el ancho de línea y disminuyendo la coherencia. Consecuentemente, el sistema $\varphi$-OTDR estará incapacitado para realizar la detección de una perturbación. Por lo tanto, en el procesamiento de los datos se descartan las mediciones realizadas cuando se presenta un salto de modo. En cambio, para una láser DFB perfectamente encadenado toda la potencia de entrada al ARFO será dirigida al puerto transmitido [15]. Este comportamiento está directamente relacionado al ancho de

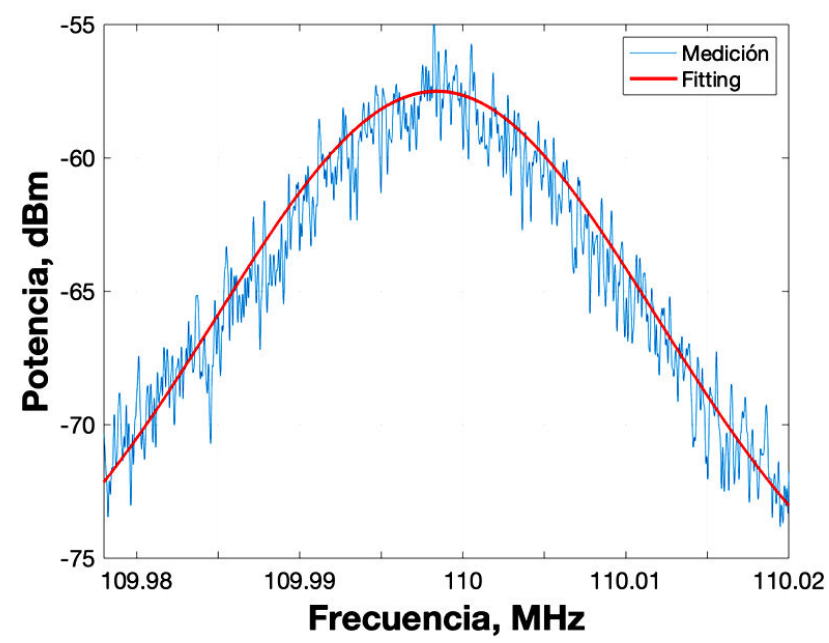

FIGURA 3. Espectro auto heterodino retardado del LEI-DFB.

línea $\Delta v$ del LEI-DFB, para su comprobación utilizamos el acoplador 99/1 del puerto C (Fig. 1), donde se monitorea el ancho de línea del LEI-DFB al modificar la potencia de la retroalimentación del puerto A. En la Fig. 2 b), se muestra la calidad del auto-encadenamiento del láser DFB en relación al ancho de línea y la potencia normalizada del puerto A. Donde se observa claramente que una disminución en la potencia del puerto A, corresponde a una reducción del ancho de línea del LEI-DFB.

El ancho de línea $\Delta v$ del LEI-DFB fue estimado mediante la medición del espectro auto-heterodino retardado [21] en el puerto C (Fig. 1). El espectro auto-heterodino retardado del láser encadenado y no encadenado fue medido empleando un interferómetro Mach-Zender desbalanceado, el retardo fue proporcionado por $40 \mathrm{~km}$ de fibra óptica en uno de sus brazos y un modulador de fase en $\sim 110 \mathrm{MHz}$ en el otro brazo. En la Fig. 3 se muestra el espectro auto-heterodino retardado del LEI-DFB en estado encadenado, su ancho de línea es reducido hasta $\Delta v=7.4 \mathrm{kHz}$ de su ancho de línea de $\sim 2 \mathrm{MHz}$ en estado no encadenado, en oscilación libre.

En nuestro experimento cada pulso lanzado en la fibra de prueba genera una traza en bruto de la señal retrodispersada de Rayleigh. Cada traza es almacenada por la tarjeta de adquisición de datos a una velocidad de adquisición de 200 Megamuestras por segundo, almacenando un total de $L=8000$ muestras correspondientes a $4.5 \mathrm{~km}$ de longitud de fibra óptica, donde la resolución por muestreo del sensor es de $\sim 0.55 \mathrm{~m}$. Para el procesamiento de la señal utilizamos $R=932$ trazas, obteniéndose una matriz $S$ de datos en bruto de $L \times R=8000 \times 932$. El procesamiento directo de la diferencia entre trazas sucesivas no permite una localización de la perturbación debido a que las bajas frecuencias producen una variación demasiado pequeña entre dos trazas sucesivas, aunque el LEI-DFB se encuentre perfectamente encadenado y en el caso que se presente un salto de modo los datos sean descartados. La Fig. 4 presenta la superposición de los valores absolutos de las diferencias consecutivas de las trazas. La señal que se genera en la posición de la perturbación en 


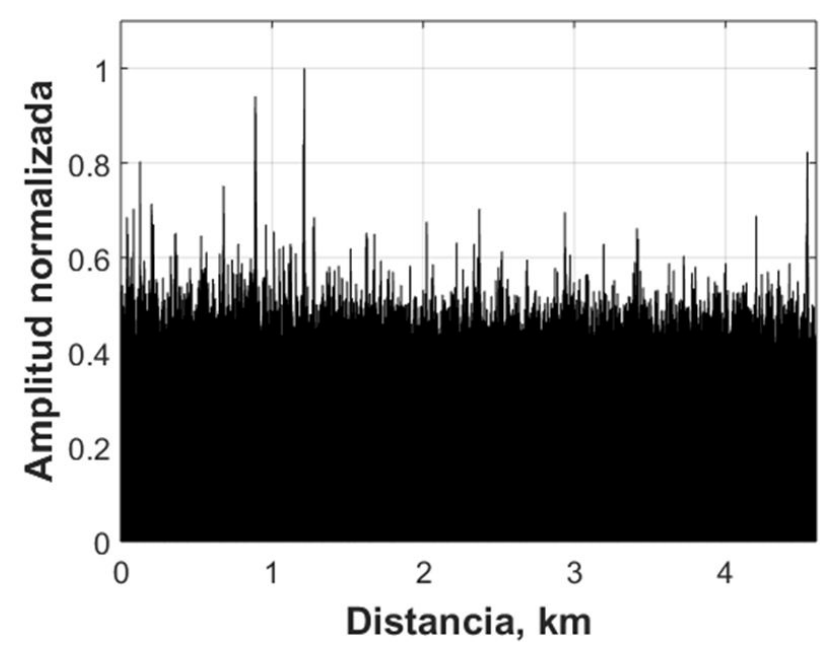

FIGURA 4. Superposición de las diferencias absolutas de las trazas consecutivas sin procesamiento, para una perturbación de $815 \mathrm{~Hz}$ en $850 \mathrm{~m}$.

$850 \mathrm{~m}$ es similar a la del ruido en una posición aleatoria, la cual alcanza una relación señal a ruido SNR estimada en $\sim 7.8 \mathrm{~dB}$.

Sin embargo, la SNR puede ser mejorada significativamente con la implementación de un algoritmo complejo de promediación espacial-temporal, el cual incluye los métodos de promediación espacial (PE) y promediación diferencial (PD).

El método de promediación espacial comprende un simple procedimiento de promediación y desplazamiento para cada $j$-trazas de renglones de la matriz $S$ :

$$
P E_{j}(l)=\frac{1}{w} \sum_{i=l-(w-1) / 2}^{l+(w-1) / 2} S_{j}(l),
$$

donde $w$ es el tamaño de la ventana de promediación, y la distancia 1 para los datos promediados se encuentra dentro del intervalo: $(w-1) / 2 \leq l \leq[L-(w-1) / 2]$.

El método de promediación diferencial (PD) comprende dos pasos, primero, para la distancia fija $l$ se debe de encontrar las sumas parciales por la promediación de $N$ elementos consecutivos de una columna de la matriz $S$ como:

$$
S_{j, m}(l)=\frac{1}{N} \sum_{i=1+m(j-1)}^{N+m(j-1)} S_{j}(l),
$$

donde $1 \leq m$ es un desplazamiento entre las sumas parciales consecutivas, y $j=1, \ldots(932-N) / m$ es un numero ordinal de la suma parcial.

Entonces se calcula el valor absoluto de la diferencia entre cada suma parcial consecutiva como:

$$
D_{k, m}(l)=\left|S_{k+1, m}(l)-S_{k, m}(l)\right|,
$$

donde $k=1, \ldots(R-N) / m-1$.

Finalmente se grafica y analiza la superposición de todas las diferencias $D_{k, m}(t)$ contra la distancia $l$. En la Fig. 5 se

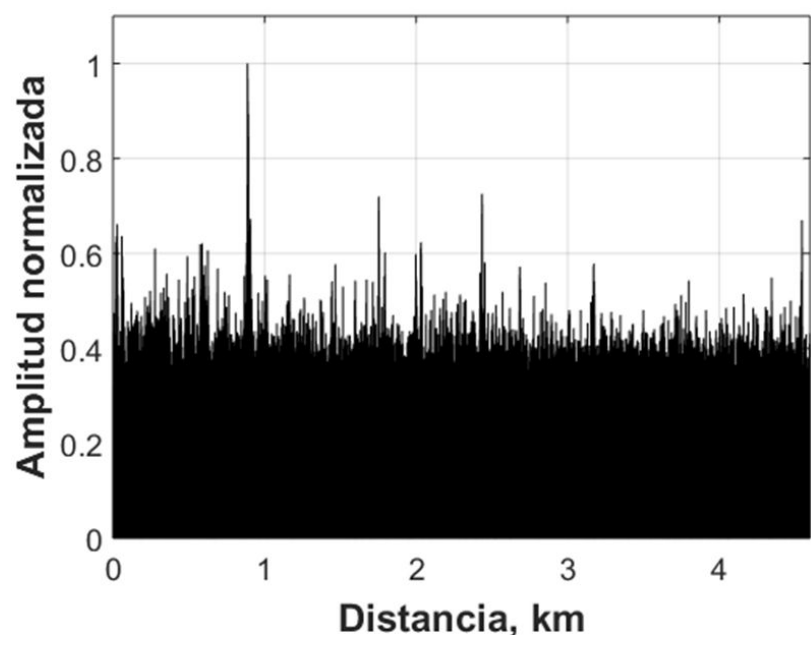

FIGURA 5. Superposición de los valores absolutos de las diferencias entre trazas consecutivas, donde $N=1$ y $m=1$, para una perturbación de $815 \mathrm{~Hz}$ en $850 \mathrm{~m}$.

muestra la superposición de los valores absolutos de las diferencias entre las trazas consecutivas para el láser DFB encadenado, con una promediación de $N=1$ y un desplazamiento de $m=1$. El pico de la señal en la distancia de $\sim 850 \mathrm{~m}$ corresponde a la perturbación generada en la fibra de prueba a una frecuencia de $815 \mathrm{~Hz}$.

El pico de la señal que sobresale es 1.4 veces mayor que el nivel promedio del ruido, para una $\mathrm{SNR}=10.7 \mathrm{~dB}$, sin embargo, la capacidad de detección puede ser mejorada considerablemente al optimizar los parámetros $N$ y $m$ en el procedimiento de la promediación. Si se encuentran los valores óptimos para los parámetros $N$ y $m$ podemos localizar un máximo valor en la SNR. Se define SNR como la relación entre la amplitud del valor absoluto de la diferencia en la distancia donde se aplica la perturbación y la amplitud promedio del ruido para todas las otras distancias: $\mathrm{SNR}=10 \mathrm{log}$ ( $\left.\mathrm{A}_{\mathrm{SEN} A \mathrm{~L}} / \mathrm{A}_{\mathrm{RUIDO}}\right)$.

En la Fig. 6 se muestra la matriz resultante de la SNR utilizando el algoritmo diferencial de promedio para diferentes

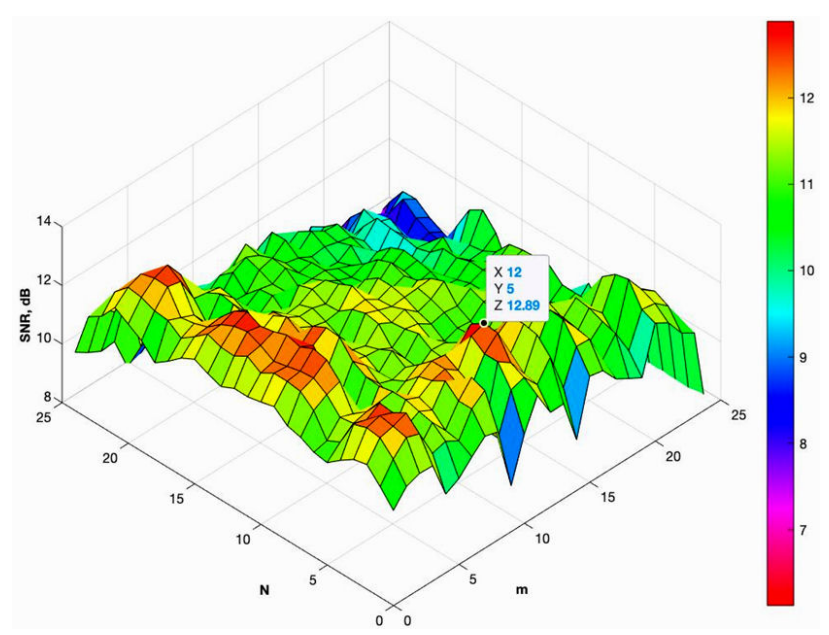

FIGURA 6. SNR para diferentes valores de $N$ y $m$, para una perturbación de $815 \mathrm{~Hz} \mathrm{a} \sim 850 \mathrm{~m}$. 


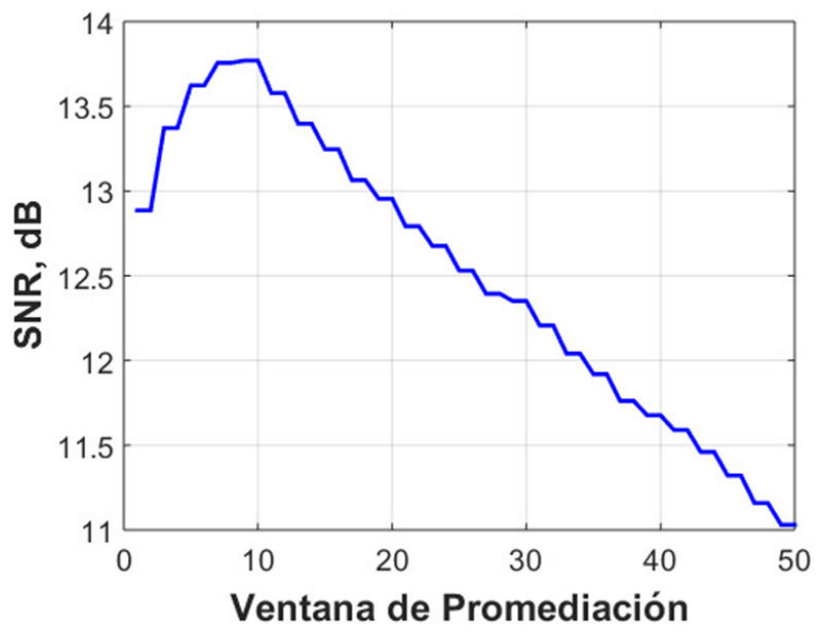

FIGURA 7. Relación señal a ruido en función del tamaño de la ventana de promediación.

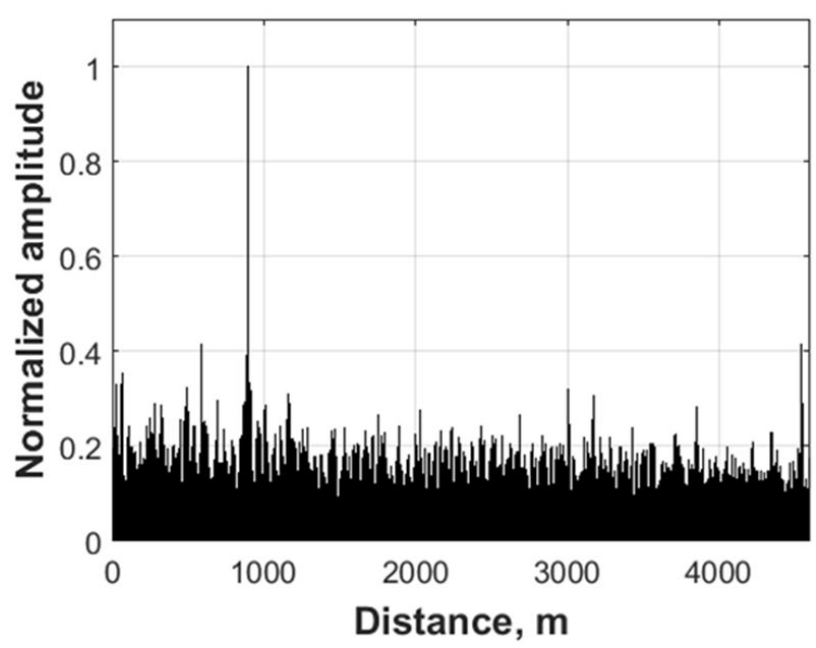

FIGURA 8. Superposición de las diferencias absolutas de las trazas consecutivas aplicando el algoritmo de procesamiento para alcanzar una SNR de $13.8 \mathrm{~dB}$.

valores de $N$ y $m$, localizando la máxima SNR de $12.9 \mathrm{~dB}$, con los parámetros óptimos de $N=5$ y $m=12$. Lo que hace posible una fiable detección y localización de una perturbación en la fibra óptica de prueba.

En la Fig. 7 se presenta la SNR contra el tamaño de la ventana de promediación para la perturbación de $815 \mathrm{~Hz}$ en una distancia de $850 \mathrm{~m}$. La máxima SNR $=13.8 \mathrm{~dB}$ es registrada para una ventana de promediación de $w=9$, donde claramente se observa que el PE con una ventana óptima de promediación incrementa la SNR $\sim 1 \mathrm{~dB}$, para alcanzar un incremento de 5-6 dB al aplicar el algoritmo propuesto.

En la Fig. 8 se muestra la superposición de las diferencias absolutas de las trazas consecutivas después de realizar el procedimiento del algoritmo de procesamiento de movimiento diferencial con los valores óptimos de $N$ y $m$, con el cual logramos un incremento significativo de la SNR de $\sim 13.8$ $\mathrm{dB}$ de la perturbación y una reducción en el promedio del ruido.

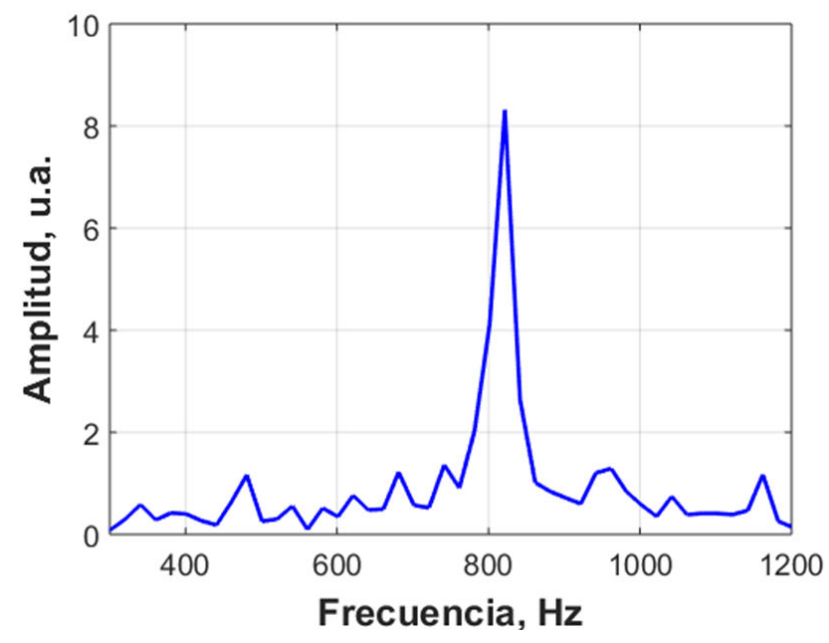

FIGURA 9. Espectro frecuencial de la perturbación adquirido a una distancia de $\sim 850 \mathrm{~m}$.

El algoritmo propuesto con el método de promediación espacial-temporal proporciona un incremento en la SNR de 3-4 dB con referencia al método diferencial usado en [18]. El valor de la SNR permite la localización correcta de la perturbación con el LEI-DFB en distancias de aproximadamente $850 \mathrm{~m}$ con una resolución de $\sim 20 \mathrm{~m}$ en una fibra de prueba de $\sim 4.5 \mathrm{~km}$. Enfatizando que cuando el láser DFB se encontraba en la condición de no encadenado, fue imposible la detección y localización de alguna perturbación en la fibra de prueba.

Al realizar un análisis suplementario de la perturbación, es posible realizar un análisis frecuencial. El espectro de frecuencia de la vibración en la distancia requerida se obtiene de la matriz de datos en crudo por medio de la transformada rápida de Fourier (FFT). En la Fig. 9 se muestra el espectro de la frecuencia de la vibración de $\sim 815 \mathrm{~Hz}$ en la posición de 850 m obtenida con el láser LEI-DFB. El pico en el espectro es 8 veces más grande que el pico del ruido más alto, lo que proporciona un adecuado reconocimiento de la frecuencia de la vibración aplicada como perturbación con una exactitud de $\sim 1 \%$.

\section{Conclusiones}

Presentamos un nuevo algoritmo que emplea un método de promediación espacial-temporal, el cual proporciona un incremento en la SNR de 5-6 dB con referencia al valor diferencial de las trazas consecutivas en bruto, aumentando significativamente la capacidad de detección de perturbaciones del sistema $\varphi$-OTDR. El método es adaptado para emplearse en un sistema que utiliza un láser DFB de bajo costo encadenado por auto-inyección a través de un anillo resonador de fibra óptica. El ancho de línea del láser DFB encadenado alcanzó $\sim 7.4 \mathrm{kHz}$, permitiendo mediciones de perturbaciones en largas distancias con el sistema $\varphi-$ OTDR.

Experimentalmente demostramos la localización de una perturbación de $815 \mathrm{~Hz}$ de frecuencia a una distancia de alrededor de $850 \mathrm{~m}$ con una exactitud de $20 \mathrm{~m}$. 


\section{Agradecimientos}

This work was supported by project N 265517 CONACyT, Mexico. Russian Science Foundation (18-12-00457) and Russian Foundation for Basic Research (18- 42732001RMK, 19-42-730009 r_a). J. L. Bueno Escobedo is sponsored by the CONACyT as Postdoctoral Fellow.
1. A. H. Hartog, An Introduction to Distributed Optical Fibre Sensors (Boca Raton, CRC Press, 2017), pp. 53-57.

2 . F. Peng et al., Ultra-long high-sensitivity $\Phi$-OTDR for high spatial resolution intrusion detection of pipelines, Opt. Express 22 (2014) 13804, https : / / doi.org/10.1364/OE . 22 . 013804

3. Y. Zhan, Q. Yu, K. Wang, F. Yang y B. Zhang, Optimization of a distributed optical fiber sensor system based on phase sensitive OTDR for disturbance detection, Sens. Rev. 35 (2015) 382, https://doi.org/10.1108/SR-12-2014-0764

4. Q. Li, C. Zhang, L. Li y X. Zhong, Localization mechanisms and location methods of the disturbance sensor based on phasesensitive OTDR, Optik 125 (2014) 2099, https://doi. org/10.1016/j.ijleo.2013.10.036.

5. R. Zinsou et al., Recent Progress in the Performance Enhancement of Phase-Sensitive OTDR Vibration Sensing Systems, Sensors 19 (2019) 1709, https://doi.org/10.3390/ s19071709.

6. Y. Lu, T. Zhu, L. Chen y X. Bao, Distributed Vibration Sensor Based on Coherent Detection of Phase-OTDR, J. Light. Technol 28 (2010) 3243, https : //doi.org/10.1109/JLT. 2010.2078798

7. Z. Wang et al., Coherent $\Phi$-OTDR based on I/Q demodulation and homodyne detection, Opt. Express 24 (2016) 853, https://doi.org/10.1364/OE.24.000853

8. M. R. Fernández-Ruiz, H. F. Martins, J. Pastor-Graells, S. Martin-Lopez y M. Gonzalez-Herraez, Phase-sensitive OTDR probe pulse shapes robust against modulation-instability fading, Opt. Lett. 41 (2016) 5756, https://doi.org/10. 1364 /OL.41.005756

9. M. R. Fernández-Ruiz, L. Costa y H. F. Martins, Distributed Acoustic Sensing Using Chirped-Pulse Phase-Sensitive OTDR Technology, Sensors 19 (2019) 4368, https: //doi .org/ $10.3390 / \mathrm{s} 19204368$

10. Z. Ma et al., High performance distributed acoustic sensor based on digital LFM pulse coherent-optical time domain reflectometer for intrapulse event, Appl. Phys. Express 13 (2020) 012016, https://doi.org/10.7567/ $1882-0786 / \mathrm{ab} 6133$

11. M. Zabihi et al., Compensation of optical path difference in heterodyne $\Phi$-OTDR systems and SNR enhancement by generating multiple beat signals, Opt. Express 27 (2019) 27488, https://doi.org/10.1364/OE.27.027488
12. A. E. Alekseev, B. G. Gorshkov, V. T. Potapov, M. A. Taranov y D. E. Simikin, Dualpulse phase-OTDR response to propagating longitudinal disturbance, Laser Phys. 30 (2020) 035107, https://doi.org/10.1088/1555-6611/ab70b0

13. J. Ohtsubo, Semiconductor Lasers, 2nd ed. (SpringerVerlag, Berlin, 2008), https://doi.org/10.1007/ 978-3-540-72650-0

14. S. Huang et al., Dual-cavity feedback assisted DFB narrow linewidth laser, Sci. Rep. 7 (2017) 1185, https://doi. org/10.1038/s41598-017-01351-w

15. C. A. López-Mercado et al., Locking of the DFB laser through fiber optic resonator on different coupling regimes, Opt. Commun. 359 (2016) 195, https://doi.org/10.1016/j. optcom.2015.09.076.

16. V. V. Spirin, C. A. López-Mercado, P. Mégret y A. A. Fotiadi, Single-mode Brillouin fiber laser passively stabilized at resonance frequency with self-injection locked pump laser, Laser Phys. Lett. 9 (2012) 377, https://doi.org/10.7452/ lapl.201110138

17. J. L. Bueno Escobedo et al., Self-injection locking of the DFB laser through an external ring fiber cavity: Application for phase sensitive OTDR acoustic sensor, Results Phys. 7 (2017) 641, https://doi.org/10.1016/j.rinp. 2017.01 .013

18. J. L. Bueno Escobedo et al., Distributed measurements of vibration frequency using phase-OTDR with a DFB laser self-stabilized through PM fiber ring cavity, Results Phys. 12 (2019) 1840, https: //doi.org/10.1016/j.rinp. 2019.02 .023

19. J. L. Bueno Escobedo et al., Self-injection locking of the DFB laser through an external ring fiber cavity: Polarization behavior, Results Phys. 6 (2016) 59, https : / / doi .org/10. $1016 / j . r i n p .2016 .01 .017$

20. D. A. Korobko, I. O. Zolotovskii, K. Panajotov, V. V. Spirin y A. A. Fotiadi, Self-injectionlocking linewidth narrowing in a semiconductor laser coupled to an external fiber-optic ring resonator, Opt. Commun. 405 (2017) 253, https : / / doi .org/ $10.1016 / j . o p t c o m .2017 .08 .040$

21. D. Derickson, Fiber Optic Test and Measurement (Prentice Hall, Upper Saddle River, 1998). 